\title{
Three-Dimensional Visualization of Ligamentum Flavum Relative to the Affected Spinal Nerve Root by MRI/CT Fusion Imaging: Three Case Reports of Lumbar Radiculopathy and Motor Palsy
}

\author{
Junji Kamogawa $^{1 *}$, Osamu Kato ${ }^{2}$ and Tatsunori Morizane ${ }^{2}$
}

${ }^{1}$ Department of Spine \& Sports Center

${ }^{2}$ Department of Radiology, Shiraishi Hospital, Ehime, Japan

\begin{abstract}
The objective of this study was to introduce a technique that enables the virtual anatomy of the ligamentum flavum (LF) to be assessed using 3D magnetic resonance (MR)/computed tomography (CT) fusion images that show the compression of the lumbar nerve root by both the herniated disc and the LF at the lateral canal zone or the foraminal zone in patients with lumbar radiculopathy or motor palsy. MR and CT images were obtained from three patients with lumbar radiculopathy with or without lower limb motor palsy. The most important characteristic evident on the images was flattening of the nerve root related to the LF, likely resulting from degenerative changes. Occasionally, the LF may play a role in root compression. In cases of motor palsy secondary to a herniated disc, the affected root was compressed with tortuosity. The flattened portion of the root seemed to change the angle of its path. In the case with lateral canal stenosis, the root was observed to be compressed between the disc and LF, resulting in constriction. In the case of degenerative lumbar scoliosis, abnormal complicated paths are seen as a result of vertebral rotation, which can change the angle of the nerve root path twice. The 3D MR/CT fusion imaging technique enhances visualization of the pathoanatomy in an otherwise blind area in the lumbar spine that is composed of the root and intervertebral foramen. Clinicians can more clearly identify the narrowest portion of the root using this 3D visualization of both the LF and disc.
\end{abstract}

Keywords: Virtual anatomy; 3D Magnetic resonance; Fusion images; Lumbar radiculopathy; Motor palsy

\section{Introduction}

Low back pain or related radiculopathy is the most common condition for which adult patients seek medical attention. In these situations, lumbar radiculopathy can result in disability and reduce health-related quality of life with irritable pain and anxiety. Two major causes of lumbar radiculopathy are disc herniation and spinal canal stenosis. The pathoanatomy of lumbar spinal canal stenosis can vary with combinations of soft or hard disc herniation, facet hypertrophy and cyst formation, and ligamentous hypertrophy, resulting in narrowing the central [1] or lateral canal [2].

The ligamentum flavum (LF) is attached to the anterior portion of the upper lamina above and both the anterior and posterior portions of the lower lamina below. It makes up the posterior wall of the spinal canal and foramina. Because it is made up of connective tissue, it affects the intrinsic stability of the spine, controls intervertebral movement, and maintains the smooth surface of the posterior dural sac [3]. Although normal ligaments are composed entirely of yellow elastic fibers and grossly have considerable elasticity like an elastic band, degenerative changes of the lumbar LF such as thickening, scarring, and calcified deposition can occur to varying degrees with age [4-6]. The thickening of the LF is considered to be an important cause of back pain or radiculopathy in lumbar degenerative disease [7-11]. Some studies $[4,5,12-14]$ have reported that the thickness of the LF in subjects with spinal stenosis varies at different spinal levels with the thickest section typically at the L4-5 level, followed by the L3-4 and L5-S1 levels. The mean thickness in most studies ranged from $3.5 \mathrm{~nm}$ to $4.5 \mathrm{~mm}$.

We reported the first 3D MR/CT fusion images of the craniovertebral junction in 2009 [15]. Since then, we have continued to develop this technique and have used it to visualize the lumbar [16-18] and cervical $[19,20]$ nerve roots. Moreover, we have shown the impingement and flattening of cervical nerve roots along with bone deformities. Patients who have experienced pain or palsy for a long duration might exhibit flattening or constrictive changes of the affected root at a preganglionic site.

Although there have been several studies of lumbar MR imaging [21-25], few have been able to identify delicate nerve compression by the LF, and none have been able to show the compressed lumbar nerve root and bony deformities in a single, $3 \mathrm{D}$ image that combines $\mathrm{CT}$ and MR images.

The purpose of this report was to introduce the virtual anatomy of the LF based on 3D MR/CT fusion images. This new image is an attempt to reveal the positional relationship between the LF and the compressed nerve root and disc because as clinicians we previously were required to mentally imagine the structure of the LF. Here, we show a new 3D image of the LF in three patients with irritable radicular pain or motor palsy of the foot using this technique.

\section{Methods}

\section{Subjects}

We describe three patients who experienced back or leg pain due to lumbar degenerative disorders. The first patient had a lumbar

*Corresponding author: Junji Kamogawa, Department of Surgery, Spine \& Sports Center, 1-5-9 Matsumoto Town, Imabari City 794-0041, Ehime, Japan, Tel: 810898324135; Fax: 810898231409; E-mail: kamogawa@mb.pikara.ne.jp

Received November 21, 2016; Accepted November 22, 2016; Published December 12, 2016

Citation: Kamogawa J, Kato O, Morizane T (2016) Three-Dimensiona Visualization of Ligamentum Flavum Relative to the Affected Spinal Nerve Root by MRI/CT Fusion Imaging: Three Case Reports of Lumbar Radiculopathy and Motor Palsy. J Pain Manage Med 2: 117.

Copyright: (c) 2016 Kamogawa J, et al. This is an open-access article distributed under the terms of the Creative Commons Attribution License, which permits unrestricted use, distribution, and reproduction in any medium, provided the original author and source are credited. 
Citation: Kamogawa J, Kato O, Morizane T (2016) Three-Dimensional Visualization of Ligamentum Flavum Relative to the Affected Spinal Nerve Root by MRI/CT Fusion Imaging: Three Case Reports of Lumbar Radiculopathy and Motor Palsy. J Pain Manage Med 2: 117.

Page 2 of 7

disc herniation with ankle palsy; the second had lumbar lateral canal stenosis with ankle palsy; and the third had severe radiculopathy secondary to degenerative lumbar scoliosis. Surgical treatment by posterior decompression under microscopy was performed in the two patients with motor palsy.

\section{Three-dimensional MR/CT fusion images}

CT images were obtained using an Asteion $4^{\circ}$ 4-row CT unit (TOSHIBA, Tochigi, Japan) with the following parameters: tube voltage, $120 \mathrm{kV}$; tube current, $260 \mathrm{~mA}$; slice thickness, $1 \mathrm{~mm}$; rotational speed, $0.75 \mathrm{~s} /$ rotation; and slice thickness for reconstruction, $0.5 \mathrm{~mm}$.

MR images were obtained using an Echelon Vega 1.5-T MR unit (HITACHI, Tokyo, Japan). The two MR imaging sequences were: Three-dimensional myelography with $1.6 \mathrm{~mm}$ slice thickness (Balanced $\mathrm{SG}^{\circ}$ and $\mathrm{RSSG}^{\circ}$ by HITACHI) with the following parameters: field-ofview (FOV), $250 \mathrm{~mm}, 384 \mathrm{~mm}$; repetition time/echo time (TR/TE), $10.4 \mathrm{~ms} / 5.2 \mathrm{~ms}, 18.0 \mathrm{~ms} / 9.2 \mathrm{~ms}$; flip angle, $45^{\circ}, 10^{\circ}$; and slice thickness for reconstruction, $1.3 \mathrm{~mm}, 1.5 \mathrm{~mm}$, respectively.

Three-dimensional MR/CT fusion images were created using the Synapse Vincent computer workstation software (versions 1 and version 3.3, FUJIFILM, Tokyo, Japan) using the following four steps, which are described by our previous reports $[16,17,20]$ in detail: 1) registration of the $\mathrm{CT}$ and MR data, 2) isolation of vertebral bodies on 3D CT images, 3) mapping of the LF, the spinal nerve roots and disc on 3D MR images, and 4) final superposition.

\section{Registration of the CT and MR data}

Both CT and MR images were obtained with the lumbar spine in a neutral position. After that, the "superposition" application of the Synapse Vincent workstation software was used to place the MR data onto the CT data automatically.

\section{Isolation of vertebral bodies on 3D CT images}

The "bony isolation" application of the Synapse Vincent" workstation software was used to automatically separate each vertebra from L1 to L5 using one click. Colors were then added to show each vertebral level easily. Using this technique, the vertebrae can be made translucent at any density.

\section{Mapping of the LF, the spinal nerve roots and disc on 3D MR images}

Two MR imaging sequences were used to obtain views of the spinal or paraspinal soft tissue: Balanced $\mathrm{SG}^{\circ}$ for cerebrospinal fluid and $\mathrm{RSSG}^{\circ}$ for the LF, nerve roots, and disc (Figure 1).

To map the path of the nerve root from the multiplanar reconstruction (MPR) view, the "center line editing" application of the Synapse Vincent" workstation software was used to place dots on the nerve roots in the MPR image. Then, curved nerves roots were converted to straight nerve roots using the curved planar reformation method, and the "center line editing" application of the Synapse Vincent "workstation software was used to identify the true long axis of the straight nerve. Next, the "contour editing" application of the workstation software was run to repair the edge of the straight nerve while rotating the root around the axis. Finally, the root was reconverted to the original shape. It took $45 \mathrm{~s}$ for one root extraction (Figure 2). RSSG

We found it to be easier to map the LF from the MPR view with

Final superposition technique was as follows: The 3D MR image was placed onto the 3D CT image enabling the entire lumbar spine to be observed. Each 3D fusion image required one hour or less of processing at the workstation by a radiological technician.
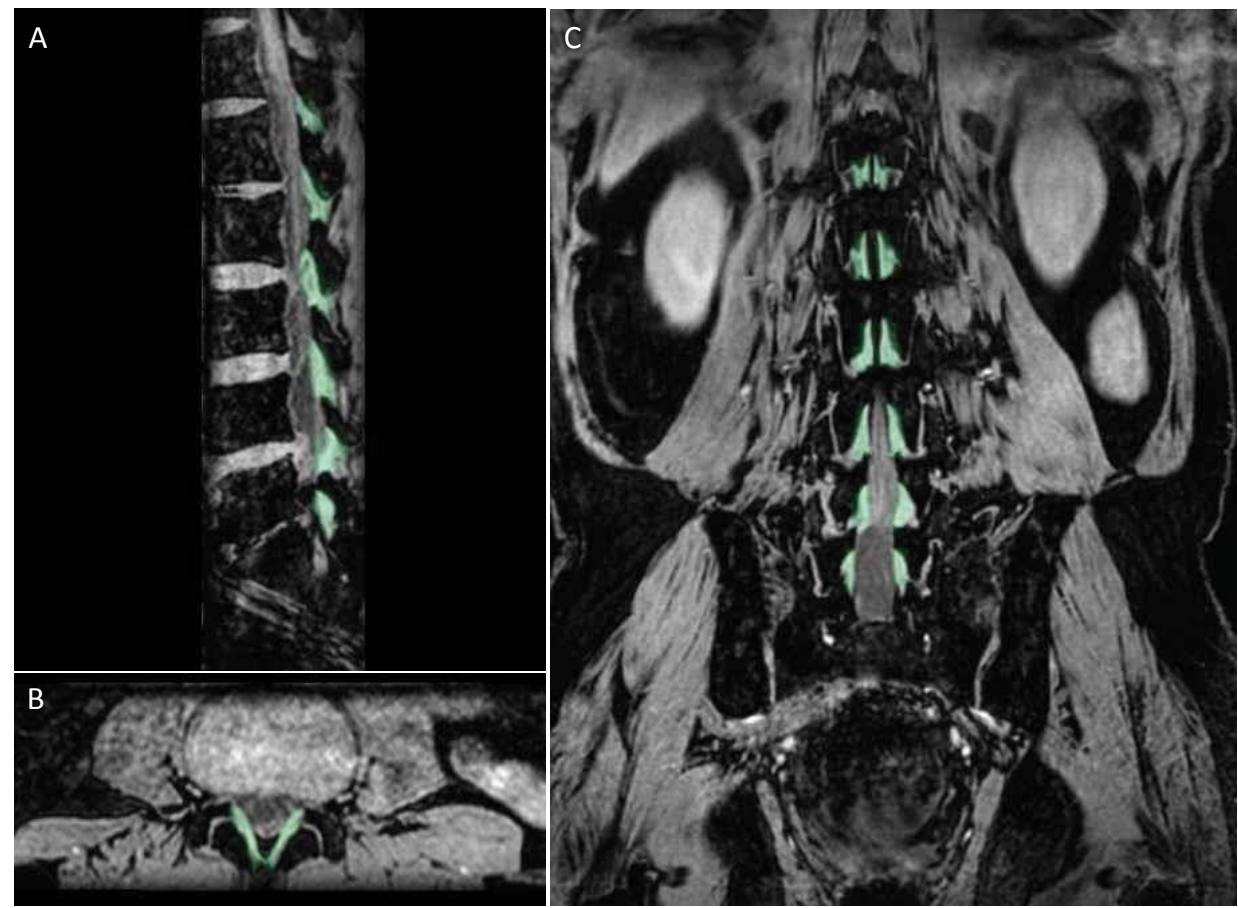

Figure 1: Magnetic resonance images showing the mapping of the ligamentum flavum on the workstation (A: sagittal; B: axial; C: frontal). The ligamentum flavum is shown in green. 

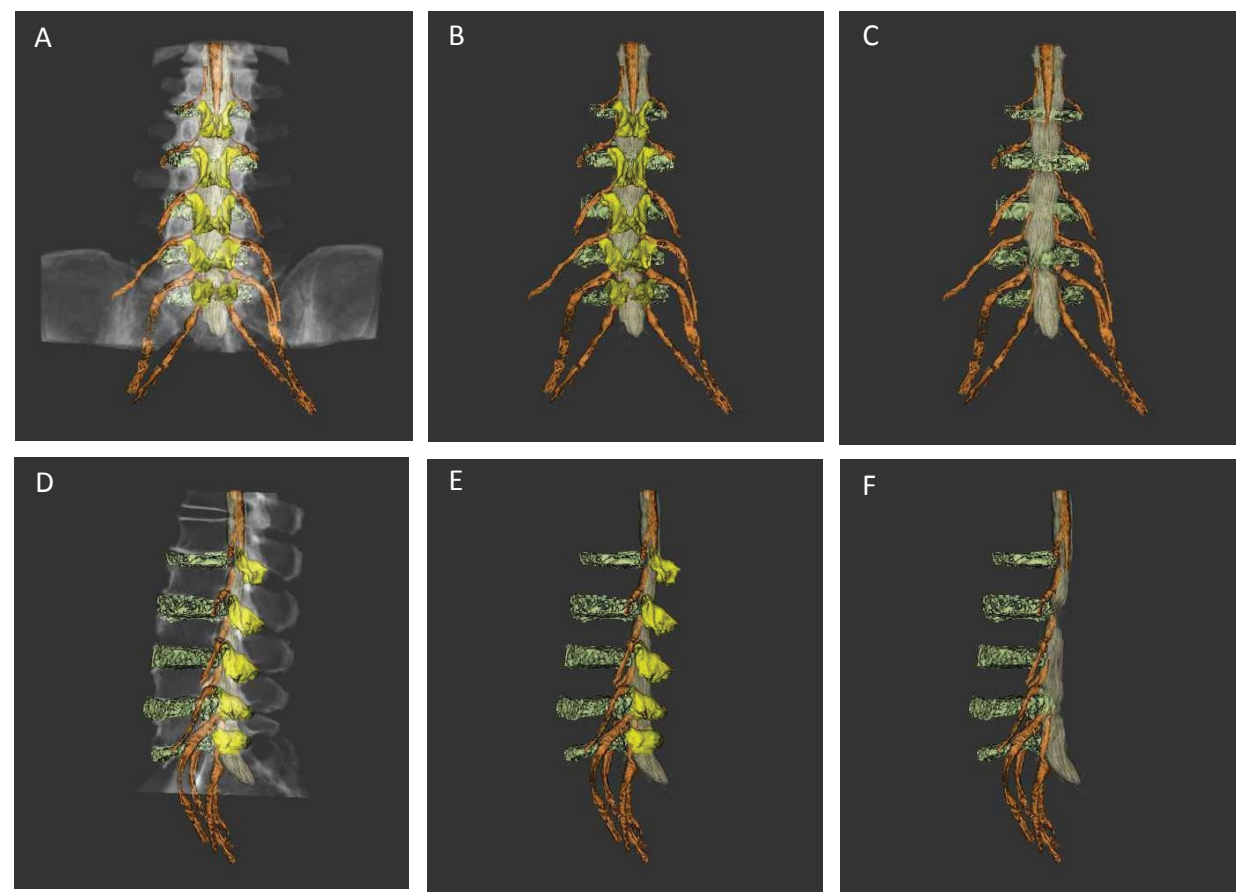

Figure 2: Three-dimensional MR/CT fusion images showing the whole lumbar spine. Images show the lumbar vertebrae and upper pelvis (A: posterior-anterior view; D: lateral view), spinal cord (orange), nerve root (orange), disc (green) and ligamentum flavum (yellow), without bone (B, E), without ligamentum flavum (C, F).

\section{Results}

\section{Case descriptions}

Case 1: A 34-year-old man who was a nurse, had a three-month history of back pain (numerical rating scale (NRS): 8) radiating into the right leg with numbness due to a herniated lumbar disc. Recently, he had developed a motor palsy of the right ankle (ankle extension, manual muscle test: 3/5). Based on the clinical symptoms and the findings on MRI, we suspected an L5 radiculopathy. Because the palsy was worsening, we proceeded with microscopic discectomy. Intraoperatively, the large extruded herniated L5-S1 disc was found to be displaced upward and was compressing both the L5 and S1 roots. Ten days after the operation, his ankle palsy completely resolved (Figure 3).

Case 2: A 59-year-old man who was a desk worker had degenerative lumbar lateral canal stenosis with L5 radiculopathy. The main clinical findings were a three-month history of severe back pain (NRS: 5) and irritable left leg pain (NRS: 9). He had recovered from back pain with conservative treatment, but subsequently unexpectedly developed a palsy of the tibialis anterior muscle (ankle extension, manual muscle test: $2 / 5)$.

We performed lumbar laminoplasty with resection of the LF under microscopy. After surgery, the radicular leg pain disappeared completely, and the motor palsy resolved in 6 months (Figure 4).

Case 3: An 82-year-old woman presented with degenerative lumbar scoliosis and syringomyelia at the level of the conus medullaris with a right L3 radiculopathy (NRS: 10) originating at the convex side of the scoliotic deformity. She had experienced back pain with irritable radicular pain of the right leg for more than six months, as well as a gait disturbance due to motor weakness and had continued to take nonsteroidal anti-inflammatory drugs. The symptoms had been getting worse, and she had developed increasing anxiety. She required a cane for ambulation, and she sometimes needed to assist her right thigh due to muscle weakness.

Selective right $\mathrm{L} 3$ root blocks were performed as both diagnostic and therapeutic tools. These reproduced the patient's pain and subsequently reduced her pain level.

After conservative treatment with four nerve root blocks, she was able to walk well and became less anxious as her pain disappeared. The fusion image guided interventions enabled us to perform selective root blocks easily and precisely (Figure 5).

\section{Discussion}

The spine is the long organ in the body and has quite a complex anatomy. The multiple nerve roots from the spinal cord follow different complex paths at each intervertebral level. There are several problems to consider when imaging in the presence of radiculopathy. First, we have traditionally routinely used the sagittal and axial spinal views in both MRI and CT. Using these methods, evaluating a limited number of cross-sectional views is not useful in identifying abnormalities along root pathways. These conventional methods may risk overlooking the root pathology. Secondly, there are four pathological spinal conditions that may disturb the ability to obtain clear imaging results, including scoliosis, vertebral rotation, spondylolisthesis, and the postoperative state. In these conditions, we cannot accurately evaluate the central spinal canal or the spinal nerve roots that are far from center with certainty. Finally, when the site of nerve compression is peripheral to the root sleeves, even myelography or CT myelography may not provide useful information.

Our technique can reveal the accurate positional relationships between the above-mentioned pathological conditions and the affected root, not only for bony problems but also for situations with postoperative epidural adhesion or extreme lateral lumbar disc 
Citation: Kamogawa J, Kato O, Morizane T (2016) Three-Dimensional Visualization of Ligamentum Flavum Relative to the Affected Spinal Nerve Root by MRI/CT Fusion Imaging: Three Case Reports of Lumbar Radiculopathy and Motor Palsy. J Pain Manage Med 2: 117.
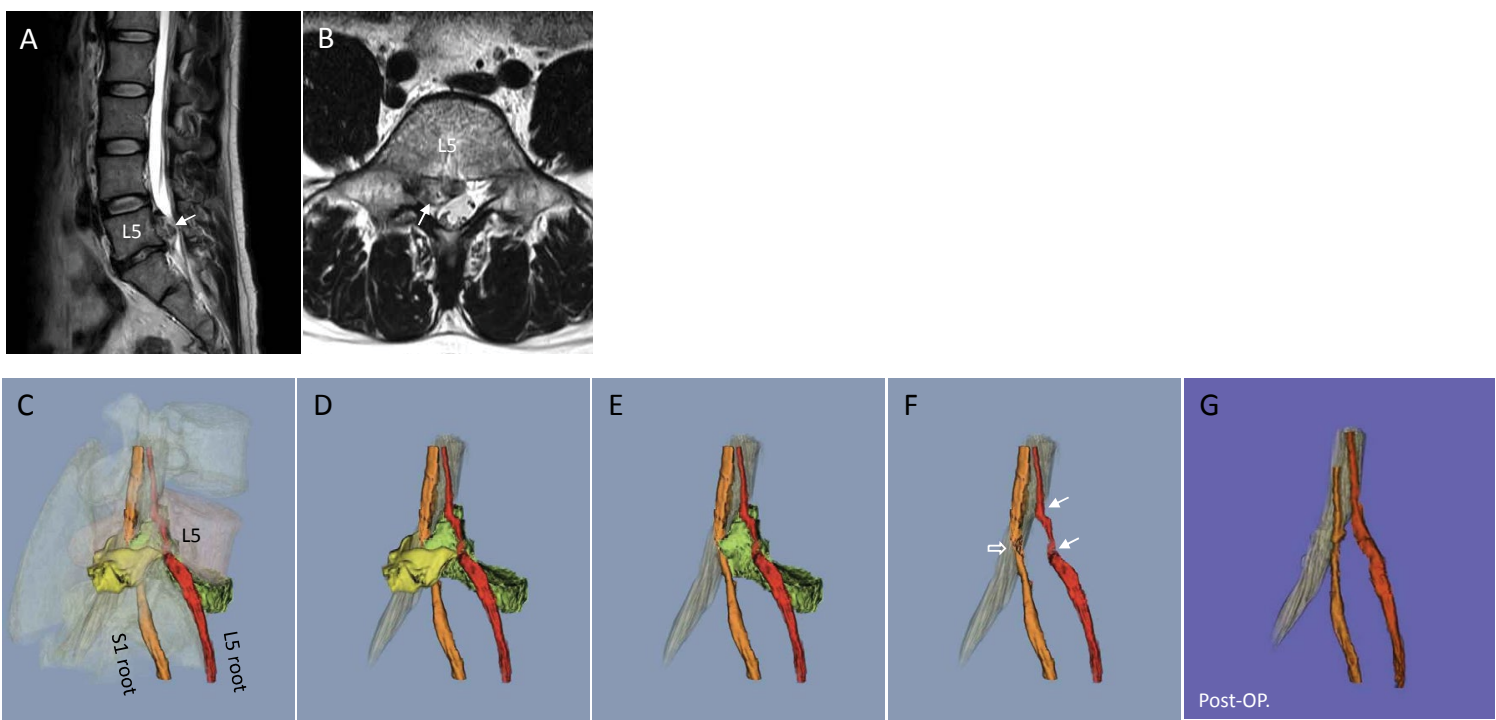

Figure 3: Flattening of the affected L5 nerve root. Large herniated disc (arrow) on the dorsal side of the L5 vertebra (A: sagittal; B: axial). Translucent bone structure $(C)$, without bone $(D)$, without ligamentum flavum $(E)$, without disc $(F)$. Postoperative view shows roots in their normal pathway and shape $(G)$.
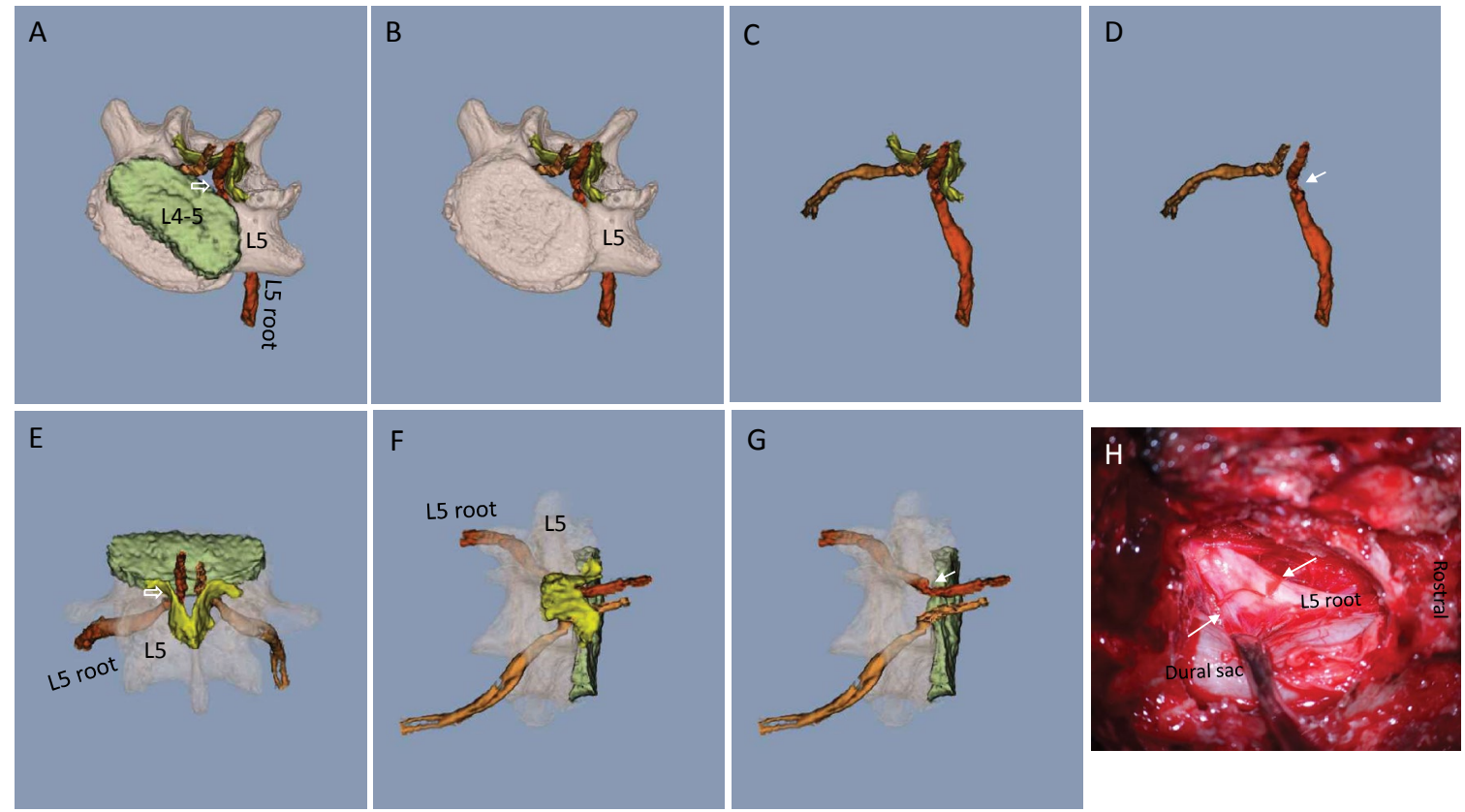

Figure 4: The 3D MRI/CT fusion image on superior-ventral observation, from within the canal (A), without disc (B), bone (C), and ligamentum flavum (D), posterior-superior view $(E)$, posterior-oblique view $(F, G)$. Images are shown with semi-translucent vertebrae $(E, F, G)$ confirming the accuracy of the fusion image by (arrow; $\mathrm{H})$.

herniation $[26,27]$. In these pathoanatomical conditions, it is easy to understand that the affected root would be difficult to see using routine methods, which has been described as the "hidden zone" by Macnab [28] and the "far-out zone" by Wiltse et al. [29].

In addition, it is quite difficult for clinicians and radiological technicians to grasp the overall shape, location, and pathology of the LF on conventional 2D MRI or even on CT myelography. The portion of the LF that is attached near the root can be especially unclear. The three major reasons for failing to detect the LF are: 1) the LF is a small and thin anatomical object; 2) there are multiple levels (bilateral L1-
S2), each with their own complicated shape; and 3) it is not possible to provide enhancement with contrast media.

In 2010, we reported 3D visualization of the LF using 256 row CT myelography in the Proceedings of the $83^{\text {rd }}$ Annual Meeting of the Japanese Orthopedic Association (unpublished data). Since then, we have secured more accurate imaging of the LF using 3D-MRI compared with that of CT. We have concluded that the LF image obtained on MRI is easier to detect using a computer workstation and provides a clearer view than CT. In this report, we demonstrated how to identify the 

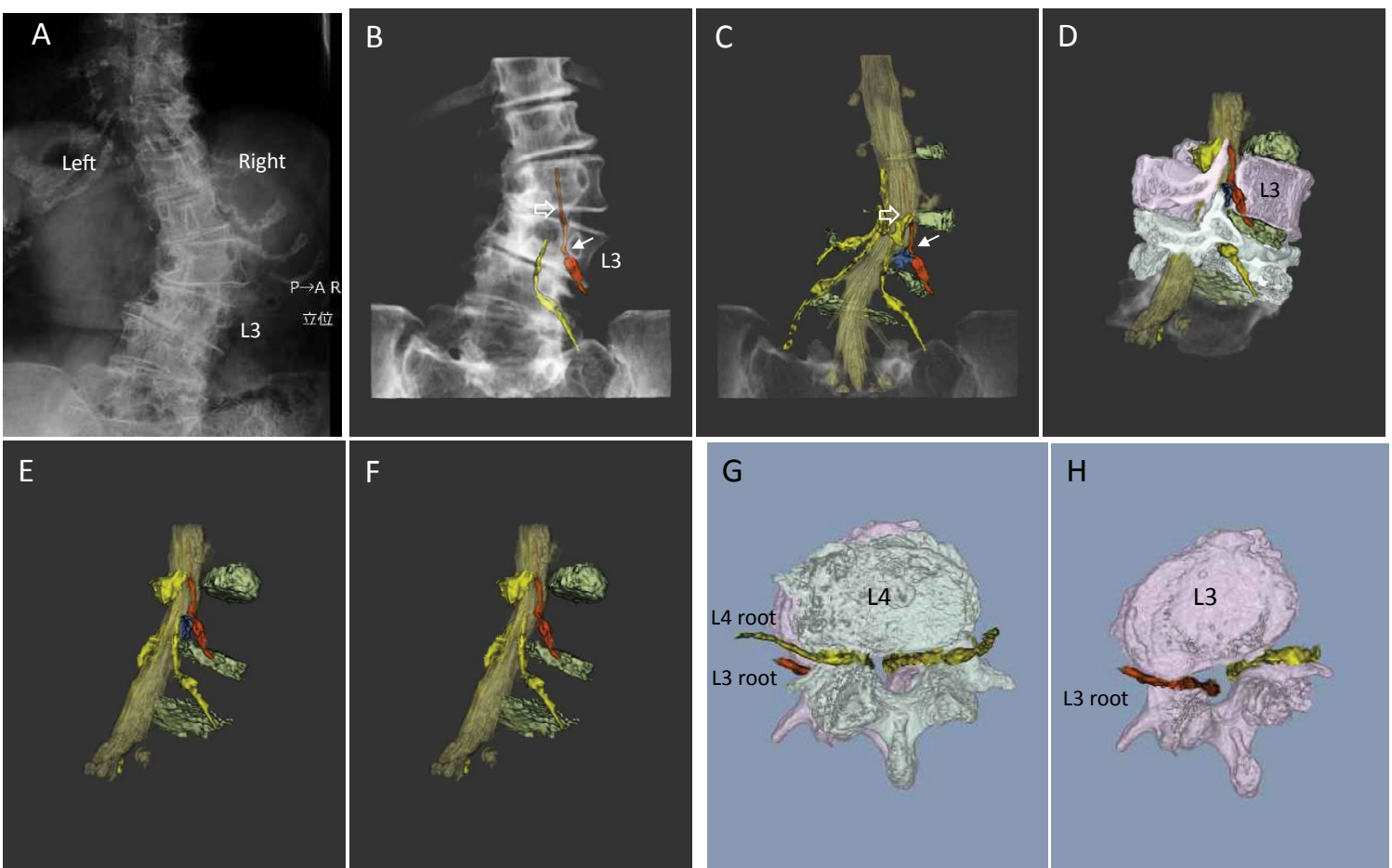

Figure 5: Plain $x$-ray with lumbar scoliosis. With a right convexity (A). Posterior view with bone (B), soft tissue and pelvis (C), right posterior-oblique view (D), without bone $(E)$, and without facet cyst $(F)$. The inferior view $(G, H)$ shows root pathway on vertebral rotation. The affected $L 3$ root is shown in red, disc is shown in green, ligamentum flavum is shown in yellow, facet cyst is shown in blue.

lumbar LF using 3D MRI as well as its positional anatomy in relation to an associated lumbar radiculopathy.

We emphasize that $3 \mathrm{D}$ visualization of the LF using this technique would be helpful for all hospital staff, not only as an evaluation method, but also as an educational tool for new physicians, nurses, therapists, radiological technicians, and medical students. Additionally, this modality would be particularly useful in pain management because in most lumbar posterior decompressive surgery without instrumentation, the main operative target may be the LF. Moreover, patients and their families would also be able to clearly view a more accurate location of the source of their pain.

Thickness or enlargement of the LF results in some causes from mechanical stress and degeneration, which are important factors in the development of radiculopathy. One possible reason for thickening is that, after rupture of some elastic fibers of the LF occurs, due to trauma or spondylolysis, repair takes place subsequently and scar tissue is formed with further enlargement of the ligaments. Another cause of morphological change in the LF is a change in lumbar spinal alignment, as a result of decreasing disc height, spondylolisthesis, scoliosis, or vertebral rotation [5], and the asymmetrical mechanical stress that the LF bears over a lifetime may lead to asymmetrical thickening $[3,14]$. Furthermore, microscopic spinal surgeons occasionally observe calcific deposition in the LF intraoperatively, which may contribute to thickening related to postoperative inflammation.

Some have reported that a decrease in disc height or disc collapse associated with disc degeneration can lead to a buckling of the LF $[5,10,30]$. In our report, we have not yet identified the pathological changes such as bulging, erosion, or a fold or tear of the LF using this technique. As there have been reports about the positional $[18,31,32]$ relationships between the LF and the dural sac and nerve root as well as detailed anatomical reports about the lateral portion of the LF [5], we will compare intraoperative views and preoperative imaging of the LF in the future.

With aging, the nerve root in degenerative scoliosis or vertebral rotation may be more vulnerable to compression by a bone spur or a thickened LF. In these malalignment conditions, our technique can be very useful in revealing the path and pathology of the affected nerve root. In particular, the clinician needs to be able to evaluate the change in the path and condition of the root resulting from traction with vertebral rotation. In that situation, the pedicle serves as a fulcrum with the pathological modification of the facet posteriorly and the disc anteriorly.

There have been several reports about morphological changes of nerve roots [21,33-38]. However, there are no direct or gold standard imaging signs that can show the accuracy of nerve root pathology because of false positive/negative MRI finding. Findings such as a transverse path or constriction of the nerve root, obscurity of the spinal ganglion, and nerve swelling have been reported as good indicators of associated symptoms [38], although morphological changes to the nerve root do not necessarily correlate with radiculopathy. We suppose that these morphological changes of the nerve root may occur as a result of bony deformities, disc bulging, thickness of the LF, facet osteophyte, or effusion. Therefore, clinicians must pay attention not only to the final finding of an abnormal root pathway or morphology as a content, but also causal degeneration changes of skeletal passageway as a container.

Although we must confirm the clinical relevance of flattening or twisted conditions of the nerve root when compared with electrophysiological tests and clinical symptoms, we think that concaved flattening or tapering, twisting with rotational traction and 
Citation: Kamogawa J, Kato O, Morizane T (2016) Three-Dimensional Visualization of Ligamentum Flavum Relative to the Affected Spinal Nerve Root by MRI/CT Fusion Imaging: Three Case Reports of Lumbar Radiculopathy and Motor Palsy. J Pain Manage Med 2: 117.

foraminal zone stenosis with central stenosis are all potential candidates for causing a motor palsy of a long duration.

However, morphological changes are not the sole cause for clinical symptoms of severe pain. In Case 3, the patient recovered from irritable pain with only a nerve root block. After conservative treatment and resolution of pain, the pathological image, i.e., the flattened or twisted condition of the nerve root would probably have the same appearance as that of pre-treatment. We suspect that there are additional causative chemical or biomechanical factors from the facet joint, disc, and epidural varix that induce the pain. In consideration of "pathoimagiology [20]", we must keep in mind that morphological changes of the nerve root would likely be one but not the only cause of the pain. Irritable radicular pain can be multifactorial.

We think that what patients fear most is the unknown origin of pain. Patients might wonder where the pain and numbness originate and whether their leg symptoms will resolve. This adds to stress and anxiety. Through accurate diagnosis supported by excellent 3D fusion images, clinicians can provide more effective education and treatment, thus decreasing anxiety and provided patients with a more hopeful future. This technique could provide new insight on imaging biomarkers for spinal pain. However, it is time consuming to produce $3 \mathrm{D}$ fusion image because different soft tissues such as nerve root, disc and LF have to be isolated. A total of $2 \mathrm{~h}$ to $3 \mathrm{~h}$ is required to produce 3D fusion image by a computer workstation. This image needs both MRI data and CT data. Therefore, it might not be implemented in routine clinical practice. We have had no imaging approach a link to the muscles or vessels of the spine so far. Although our technique is associated with problems such as convenience, misalignment between the two modalities and applicability, we intend to continue with further development in the future.

\section{Conclusion}

This study introduced new technique enables 3D anatomy of lumbar ligamentum flavum using 3D MR/CT fusion images. Anyone can understand the affected nerve root compression. Pain clinicians and spinal surgeons can use 3D MR/CT fusion images as pre-treatment maps and for intraoperative navigation. In addition, the 3D MR/CT fusion images can be used as educational materials for hospital staff and also for patients and their families who will benefit from a better understanding of the path anatomy before providing informed consent for treatments.

\section{Ethical Standards and Patient Consent}

We declare that all human and animal studies have been approved by the Japanese Orthopedic Association and have therefore been performed in accordance with the ethical standards laid down in the 1964 Declaration of Helsinki and its later amendments. We declare that all patients gave informed consent prior to inclusion in this study. And we have obtained the patient's consent to publish in this paper.

\section{References}

1. Tomkins-Lane CC, Battié MC, Hu R, Macedo L (2014) Pathoanatomical characteristics of clinical lumbar spinal stenosis. J Back Musculoskelet Rehabil 27: 223-229.

2. Nowicki BH, Haughton VM, Schmidt TA, Lim TH, An HS, et al. (1996) Occult lumbar lateral spinal stenosis in neural foramina subjected to physiologic loading. AJNR Am J Neuroradiol 17: 1605-614

3. Safak AA, Is M, Sevinc O, Barut C, Eryoruk N, et al. (2010) The thickness of the ligamentum flavum in relation to age and gender. Clin Anat 23: 79-83.

4. Altinkaya N, Yildirim T, Demir S, Alkan O, Sarica FB (2011) Factors associated with the thickness of the ligamentum flavum: is ligamentum flavum thickening due to hypertrophy or buckling? Spine (Phila Pa 1976) 15: E1093-E1097.

5. Okuda T, Fujimoto Y, Tanaka N, Ishida O, Baba I, et al. (2005) Morphological changes of the ligamentum flavum as a cause of nerve root compression. Eur Spine J 14: 277-286.

6. Twomey L, Taylor J (1988) Age changes in the lumbar spinal and intervertebral canals. Paraplegia 26: 238-249.

7. Hasegawa T, An HS, Haughton VM, Nowicki BH (1995) Lumbar foraminal stenosis: critical heights of the intervertebral discs and foramina. A cryomicrotome study in cadavera. J Bone Joint Surg Am 77: 32-38.

8. Kirkaldy-Willis WH, Wedge $\mathrm{JH}$, Yong-Hing K, Reilly J (1978) Pathology and pathogenesis of lumbar spondylosis and stenosis. Spine (Phila Pa 1976) 3: 319-328.

9. Panjabi MM, Takata K, Goel VK (1983) Kinematics of lumbar intervertebral foramen. Spine (Phila Pa 1976) 8: 348-357.

10. Postacchini F, Gumina S, Cinotti G, Perugia D, DeMartino C (1994) Ligamenta flava in lumbar disc herniation and spinal stenosis. Light and electron microscopic morphology. Spine (Phila Pa 1976) 15: 917-922.

11. Ramani PS, Perry RH, Tomlinson BE (1975) Role of ligamentum flavum in the symptomatology of prolapsed lumbar intervertebral discs. J Neurol Neurosurg Psychiatry 38: 550-557.

12. Abbas J, Hamoud K, Masharawi YM, May H, Hay O, et al. (2010) Ligamentum flavum thickness in normal and stenotic lumbar spines. Spine (Phila Pa 1976) 35: $1225-1230$.

13. Abdel-Meguid EM (2008) An anatomical study of the human lumbar ligamentum flavum. Neurosciences (Riyadh) 13: 11-16.

14. Kolte VS, Khambatta S, Ambiye MV (2015) Thickness of the ligamentum flavum: correlation with age and its asymmetry-an magnetic resonance imaging study. Asian Spine J 9: 245-253.

15. Kamogawa J, Katagi R, Kodama K, Shiraishi S, Yamamoto H (2009) The 3D-MRI/MRA/CT Fusion Imaging in spine and spinal cord disorders focusing on upper cervical spine: Report of two cases. Spine Spinal Cord 22: 979-982.

16. Yamanaka Y, Kamogawa J, Katagi R, Kodama K, Misaki H, et al. (2010) 3-D MRI/CT fusion imaging of the lumbar spine. Skeletal Radiol 39: 285-288.

17. Kamogawa J, Kato O (2012) Virtual Anatomy of Spinal Disorders by 3D MRI/ CT Fusion Imaging. In: Grivas TB, editor. Recent Advances in Scoliosis. InTech, Rijeka, Croatia.

18. Misaki H, Kamogawa J, Katagi R, Kodama K, Shiraishi S, et al. (2009) Image assessment of the neural impairment at the adjacent level after lumbar decompression surgery. A case report with new trial using 3D-MRI/CT Fusion Imaging. Spine Spinal Cord 22: 1171-1175.

19. Kamogawa J, Hato T, Kato O, Morizane T (2014) 3-D MRI/CT Fusion Imaging of cervical radiculopathy: two case reports. J Clin Med Imabari Med Assoc 26 1-6.

20. Kamogawa J, Kato O, Morizane T, Hato T (2015) Virtual pathology of cervical radiculopathy based on 3D MR/CT fusion images: impingement, flattening or twisted condition of the compressed nerve root in three cases. Springerplus 12: 123 .

21. Aota Y, Niwa T, Yoshikawa K, Fujiwara A, Asada T, et al (2007) Magnetic resonance imaging and magnetic resonance myelography in the presurgical diagnosis of lumbar foraminal stenosis. Spine (Phila Pa 1976) 32: 896-903.

22. Byun WM, Jang HW, Kim SW (2012) Three-dimensional magnetic resonance rendering imaging of lumbosacral radiculography in the diagnosis of symptomatic extraforaminal disc herniation with or without foraminal extension. Spine (Phila Pa 1976) 37: 840-844

23. Chang HS, Zidan I, Fujisawa N, Matsui T (2011) Microsurgical posterolatera transmuscular approach for lumbar foraminal stenosis. J Spinal Disord Tech 24: 302-307.

24. Eguchi Y, Ohtori S, Orita S, Kamoda H, Arai G, et al. (2011) Quantitative evaluation and visualization of lumbar foraminal nerve root entrapment by using diffusion tensor imaging: preliminary results. AJNR Am J Neuroradiol 32 1824-1829.

25. Heo DH, Lee MS, Sheen SH, Cho SM, Cho YJ, et al. (2009) Simple oblique lumbar magnetic resonance imaging technique and its diagnostic value for extraforaminal disc herniation. Spine (Phila Pa 1976) 34: 2419-2423. 
Citation: Kamogawa J, Kato O, Morizane T (2016) Three-Dimensional Visualization of Ligamentum Flavum Relative to the Affected Spinal Nerve Root by MRI/CT Fusion Imaging: Three Case Reports of Lumbar Radiculopathy and Motor Palsy. J Pain Manage Med 2: 117.

26. Abdullah AF, Ditto EW, Byrd EB, Williams R (1974) Extreme-lateral lumbar discherniations. Clinical syndrome and special problems of diagnosis. J Neurosurg 41: 229-234.

27. Postacchini F, Montanaro A (1979) Extreme lateral herniations of lumbar disks. Clin Orthop Relat Res: 222-227.

28. Macnab I (1971) Negative disc exploration. An analysis of the causes of nerveroot involvement in sixty-eight patients. J Bone Joint Surg Am 53: 891-903.

29. Wiltse LL, Guyer RD, Spencer CW, Glenn WV, Porter IS (1984) Alar transverse process impingement of the L5 spinal nerve: the far-out syndrome. Spine (Phila Pa 1976) 9: 31-41.

30. Schönström NR, Hansson TH (1991) Thickness of the human ligamentum flavum as a function of load: an in vitro experimental study. Clin Biomech 6: 19-24.

31. Avrahami E, Wigler I, Stern D, Caspi D, Yaron M (1990) Computed tomographic (CT) demonstration of calcification of the ligamenta flava of the lumbosacral spine associated with protrusion of the intervertebral disc. Spine (Phila $\mathrm{Pa}$ 1976) 15: 21-23

32. Hasue M, Kikuchi S, Sakuyama Y, Ito T (1983) Anatomic study of the interrelation between lumbosacral nerve roots and their surrounding tissues. Spine (Phila Pa 1976) 8: 50-58.
33. Jenis LG, An HS (2000) Spine update. Lumbar foraminal stenosis. Spine (Phila Pa 1976) 25: 389-394

34. Kunogi J, Hasue M (1991) Diagnosis and operative treatment of intraforamina and extraforaminal nerve root compression. Spine (Phila Pa 1976) 16: 1312 1320.

35. Matsumoto M, Chiba K, Nojiri K, Ishikawa M, Toyama Y, et al. (2002) Extraforaminal entrapment of the fifth lumbar spinal nerve by osteophytes of the lumbosacral spine: anatomic study and a report of four cases. Spine (Phila Pa 1976) 15: E169-E173

36. Olsewski JM, Simmons EH, Kallen FC, Mendel FC (1991) Evidence from cadavers suggestive of entrapment of fifth lumbar spinal nerves by lumbosacral ligaments. Spine (Phila Pa 1976) 16: 336-347

37. Transfeldt EE, Robertson D, Bradford DS (1993) Ligaments of the lumbosacra spine and their role in possible extraforaminal spinal nerve entrapment and tethering. J Spinal Disord 6: 507-512.

38. Yamada H, Terada M, Iwasaki H, Endo T, Okada M, et al. (2015) Improved accuracy of diagnosis of lumbar intra and/or extra-foraminal stenosis by use of three-dimensional MR imaging: comparison with conventional MR imaging. J Orthop Sci 20: 287-294. 